\title{
Expression profiles and biological implications of plasma miRNAs associated with chronic myeloid leukemia phases
}

\author{
Ji Zhang ${ }^{1,2}$, Yawen Jiang ${ }^{1}$, Xu Han ${ }^{1}$, Wenen Liu ${ }^{3}$, Xielan Zhao ${ }^{4}$ and Jing Liu ${ }^{1}$ \\ ${ }^{1}$ Molecular Biology Research Center, School of Life Sciences, Central South University, Changsha 410078, China \\ ${ }^{2}$ Department of Hematology, The First Affiliated Hospital, University of South China, Hengyang 421001, China \\ ${ }^{3}$ Department of Clinical Laboratory, Xiangya Hospital of Central South University, Changsha, 410008, China \\ ${ }^{4}$ Department of Hematology, Xiangya Hospital of Central South University, Changsha, 410008, China \\ Correspondence to: Jing Liu, email: jingliucsu@hotmail.com, liujing2@sklmg.edu.cn \\ Ji Zhang, email: zhang_ji001@hotmail.com
}

Keywords: plasma miRNA; chronic myeloid leukemia; expression profile; biological function; miR-451a

Received: September 13, 2017 Accepted: November 16, $2017 \quad$ Published: January 02, 2018

Copyright: Zhang et al. This is an open-access article distributed under the terms of the Creative Commons Attribution License 3.0 (CC BY $3.0)$, which permits unrestricted use, distribution, and reproduction in any medium, provided the original author and source are credited.

\section{ABSTRACT}

microRNAs play important regulatory roles in hematologic malignancies, and dysregulation of circulating miRNAs serves as diagnostic, prognostic and predictive biomarkers in many diseases. The correlation of plasma miRNA profiles with the progression of chronic myeloid leukemia (CML) has not been investigated. We have applied microarrays to identify differentially expressed miRNAs, followed by qRTPCR to validate candidate miRNAs from four sample pools including chronic phase (CP), accelerated phase(AP), blast crisis(BC) and healthy control. Clustering analyses revealed varying phase-specific patterns of plasma miRNA expression during CML progression. Different phases of CML showed differential expression profiles between sample pools during the progression. The functional annotation tool, DAVID, found that putative targets of dysregulated miRNAs in different phases of diease are involved in several important signaling pathways. Gradually decreased expression levels of miR-451a were validated in CML patients from the CP to AP and BC; when CML patients achieved major molecular response (MMR), miR-451a expression was increased and restored to normal range. These data provide an important resource for studies on CML progression and allow a better understanding of the fundamental differences in plasma miRNA expression profiles among the different phases of CML.

\section{INTRODUCTION}

Chronic myeloid leukemia (CML) is a kind of hematopoietic malignancy characterized by a reciprocal chromosomal translocation, $\mathrm{t}(9 ; 22)(\mathrm{q} 34 ; \mathrm{q} 11)$, known as the Philadelphia $(\mathrm{Ph})$ chromosome, producing the Bcr$\mathrm{Abl}$ oncogene $[1,2]$. CML is a multiphase disease with the phases include chronic phase (CP), accelerated phase (AP) and blast phase, also known as blast crisis (BC), and can be diagnosed in each of the three distinct phases. Most newly diagnosed CML patients are in the chronic phase [3]. CML usually progresses from the chronic phase (CP) to the accelerated phase (AP) and blast crisis (BC) with an accumulation of other cytogenetic abnormalities. It has been reported that the dysregulation of microRNAs
(miRNAs) had an important impact on the initiation, progression and drug-resistance in CML [4-6].

miRNAs are an abundant class of regulatory noncoding single-stranded RNA molecules, which are approximately 20 23 nucleotides in length. miRNAs can negatively regulate gene expression through partial base-pairing with the 3'-untranslated region of their target mRNAs, and they inhibit the translation and/ or lead to degradation of these mRNAs. Recently, more attention has been paid on the roles of circulating miRNAs in physiological and pathological processes. These circulating miRNAs are stable and can be protected from degradation by encapsulation in lipid vesicles including microvesicles, exosomes, and apoptotic bodies. Circulating miRNAs have a regulatory role in 
immune-modulation, angiogenesis, pathogenesis and progression of cancers [7-10]. Dysregulated circulatory miRNAs have been served as biomarkers for diagnosis, prognosis and prediction in hematopoietic neoplasias [11]. In recent years, several studies have reported that circulating miRNAs were involved in pathogenesis and therapy prediction in CML patients. Exosomal miR-126 in endothelial cells modulates the adhesive and migratory abilities of CML cells [12]. Patients with a low plasma miR-215 expression had a significantly higher total imatinib intake compared to patients with increased miR215 expression [13]. miR-21 was enriched in exosomes released by K562 and LAMA84 cells after treatment with curcumin, suggesting that selective packaging of miR-21 in the exosomes may contribute to the antileukemic effect of curcumin in CML [14].

Advanced phases of CML are often accompanied by multiple genetic abnormalities. CML patients with AP/BC own enhanced cell proliferation, attenuated apoptosis, blocked differentiation and some other chromosomal abnormalities and molecules, except the $\mathrm{Ph}$ chromosome and the Bcr-Abl oncogene. Dysregulated miRNAs may play important roles in CML pathogenesis and progression from $\mathrm{CP}$ to $\mathrm{AP}$ and $\mathrm{BC}$. It has been suggested that circulating miRNAs served as potential biomarkers in various diseases. However, the expression profile and biological implications of circulating miRNA have not yet been determined in CML. In this study, we used microarrays to detect the plasma miRNA expression profiles in CML. To better understand the progression of CML, we investigated the dynamics of plasma miRNA levels between different phases, and revealed its biological implications. Moreover, plasma miR-451a expression was found to decrease dramatically in different phases of CML. miR-451a may have regulatory roles in pathogenesis and imatinib resistance in patients with CML [15-17]. To further examine the possible roles of miRNAs in CML patients, we investigated plasma miRNA expression profiles at different stages of CML, which may shed light on the mechanisms involved in the progression of CML.

\section{RESULTS}

\section{miRNA expression patterns of CML during different phases}

To analyze the expression profiles of the plasma miRNAs in CML, we compared the microarray data from the different stages of CML during disease progression (Figure 1). Expression profiles of plasma miRNAs in the four sample pools were detected by miRNA microarray. We estimated the number of plasma miRNAs detected at different phases of CML. There were 302 differentially expressed miRNAs including 88 down-regulated and 214 up-regulated miRNAs between the CP and healthy control, 222 differentially expressed miRNAs including 66 up-regulated and 156 down-regulated miRNAs between the AP and CP, and 115 differentially expressed miRNAs including 48 up-regulated and 67 downregulated miRNAs between the $\mathrm{BC}$ and $\mathrm{AP}$ (Figure 2). The number of differential miRNAs between the CP and normal controls was larger than that between the AP and $\mathrm{CP}$ and the BC and AP. Moreover, 76 dysregulated miRNAs including miR-451a, miR-16-5p, and miR92a-3p showed differential expression patterns between the two sample pools during the progression of $\mathrm{CML}$ (Figure 3).

\section{Validation of microarray data and plasma sample pools}

Four down-regulated miRNAs (miR-122-5p, miR-16-5p, miR-451a and miR-92a-3p), two up-regulated miRNAs (miR-4644 and miR-6075) with more than a 2.0fold change in their expression, and two stably expressed miRNAs (miR-4656 and miR-5739) among sample pools were chosen to evaluate the microarray data and pooling precision by qRT-PCR. The heatmaps showed comparable results (Figure 4). Comparing the microarray and the qRTPCR data, we found that hierarchical clustering formed similar gene and sample clusters. However, there were some discrepancies in the data between the microarray and qRT-PCR. For example, in the normal control pool, the relative expression of miR-92a-3p detected by microarray was higher than that detected by qRT-PCR, however, regardless of the detection by microarray or PCR, the relative expression level of miR-92a-3p were consistent among these phases of CML.

\section{Differential expression patterns of miRNAs and functional annotation}

We analyzed the expression profiles of all differentially expressed plasma miRNAs during disease progression (Figure 5). The putative target genes were mediated by dysregulated miRNAs among the different phases of CML. There were 214 up-regulated miRNAs and 88 down-regulated miRNAs in the CP sample pool compared with the healthy control pool. The target genes of 302 differentially expressed miRNAs were analyzed by functional annotation, and found to play important roles in several biological processes $(p<0.0001)$. The putative targets were primarily involved in regulating signal transduction, multicellular organismal development, transmembrane transport, apoptosis and cell adhesion. 66 miRNAs were significantly increased, and 88 miRNAs were significantly down-regulated in the AP sample pool compared with the CP sample pool. The putative target genes mediated by differentially expressed miRNA between the $\mathrm{CP}$ and AP pools were involved mainly in 
regulating signal transduction, multicellular organismal development, transmembrane transport and apoptosis. Moreover, there were 48 up-regulated miRNAs and 67 down-regulated miRNAs in the BC sample pool compared with the AP sample pool. These putative targets mediated by differentially expressed miRNAs participated primarily in biological processes involved in signal transduction, regulation of transcription, DNA-dependent processes, multicellular organismal development and transmembrane transport.

We further analyzed the expression patterns of all dysregulated miRNAs across the disease progression. Based on the expression patterns of the different CML phases, the differentially expressed miRNAs clustered into four major groups, demonstrated in a heat map and graphical format (Figure 6). Two patterns revealed lowto mid-range levels of expression in the healthy samples and increased during disease progression (groups 1 and 3 ), while two other patterns showed higher levels of expression in the healthy samples and decreased distinctly during disease progression (groups 2 and 4).

We performed Gene Ontology (GO) analysis of the putative target genes, which were mediated by differentially expressed miRNAs within these four clusters. We also identified target genes enriched by GO terms to gain insight into the biological processes regulated across the disease progression (Figure 6).

\section{Validation of plasma miR-451a expression in CML by qRT-PCR}

Dramatic down-regulation of miR-451a in the progression of CML prompted us to determine expression patterns in a larger cohort of samples. To validate expression of miR-451a in CML patients, plasma samples were obtained from 20 healthy controls, $40 \mathrm{CP}$ samples, $18 \mathrm{AP}$ and BC samples and $20 \mathrm{MMR}$ samples. Because it was very difficult to collect a large cohort of $\mathrm{AP}$ and $\mathrm{BC}$ patients, we incorporated them into a group, as previously reported [18]. We detected the expression of miR-451a by qRT-PCR, and the miR-451a expression was significantly lower in CML compared with the healthy controls. Furthermore, the expression level of miR-451a decreased gradually with the development of CML, and the level of miR-451a was significantly down-regulated in the AP and BC phases, as shown in Figure 7. However, when CML patients obtained MMR after treatment, the expression level of miR-451a was

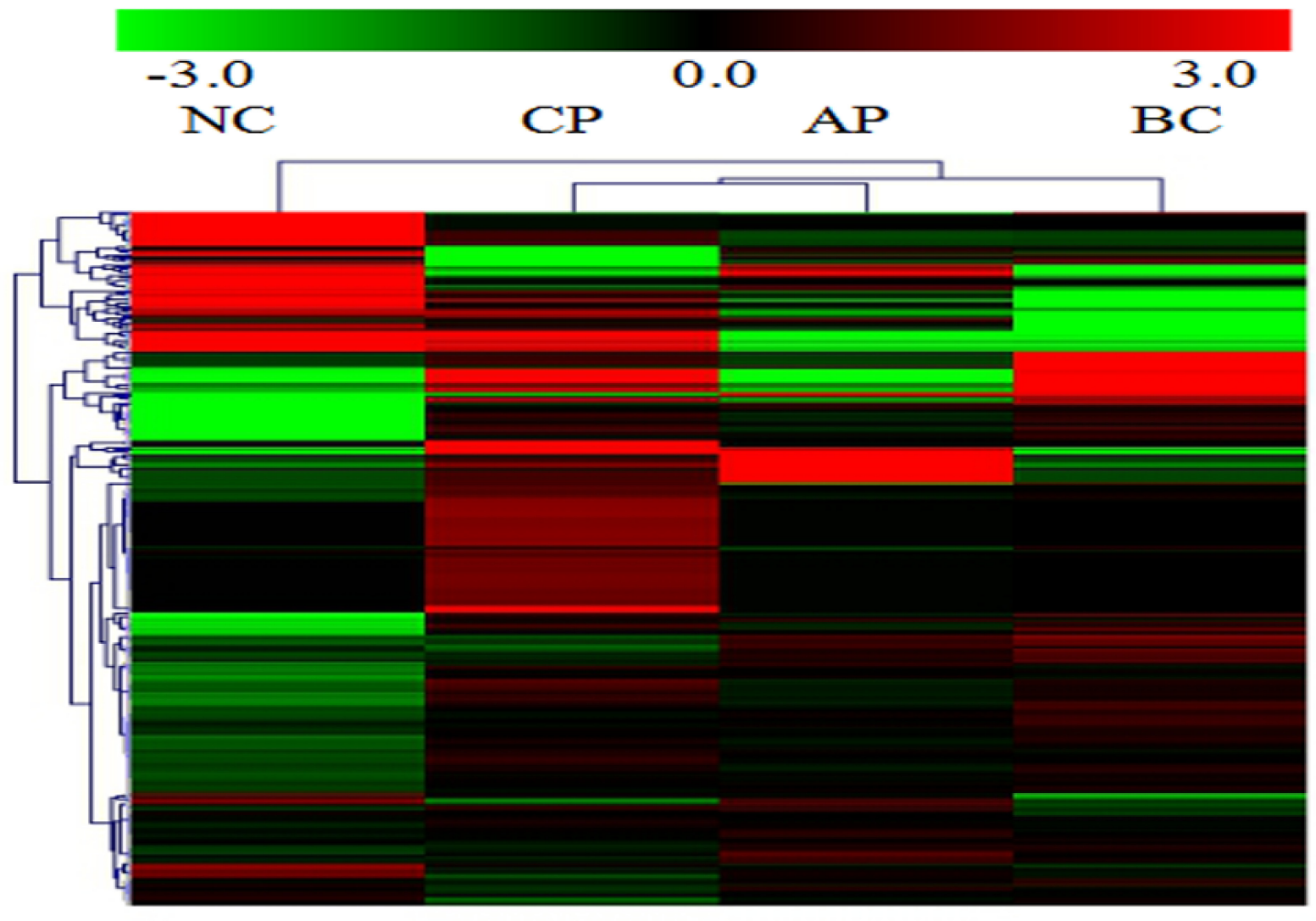

Figure 1: Global differential expression of plasma miRNA at different phases of CML. Differential expression of plasma miRNAs were detected in the four sample pools by miRNA microarray. Genespring software was used to finish the basic analysis of the raw data. The raw data was normalized with the quantile algorithm. The threshold value for up- and down-regulated genes was a fold change $\geq 2.0$. 


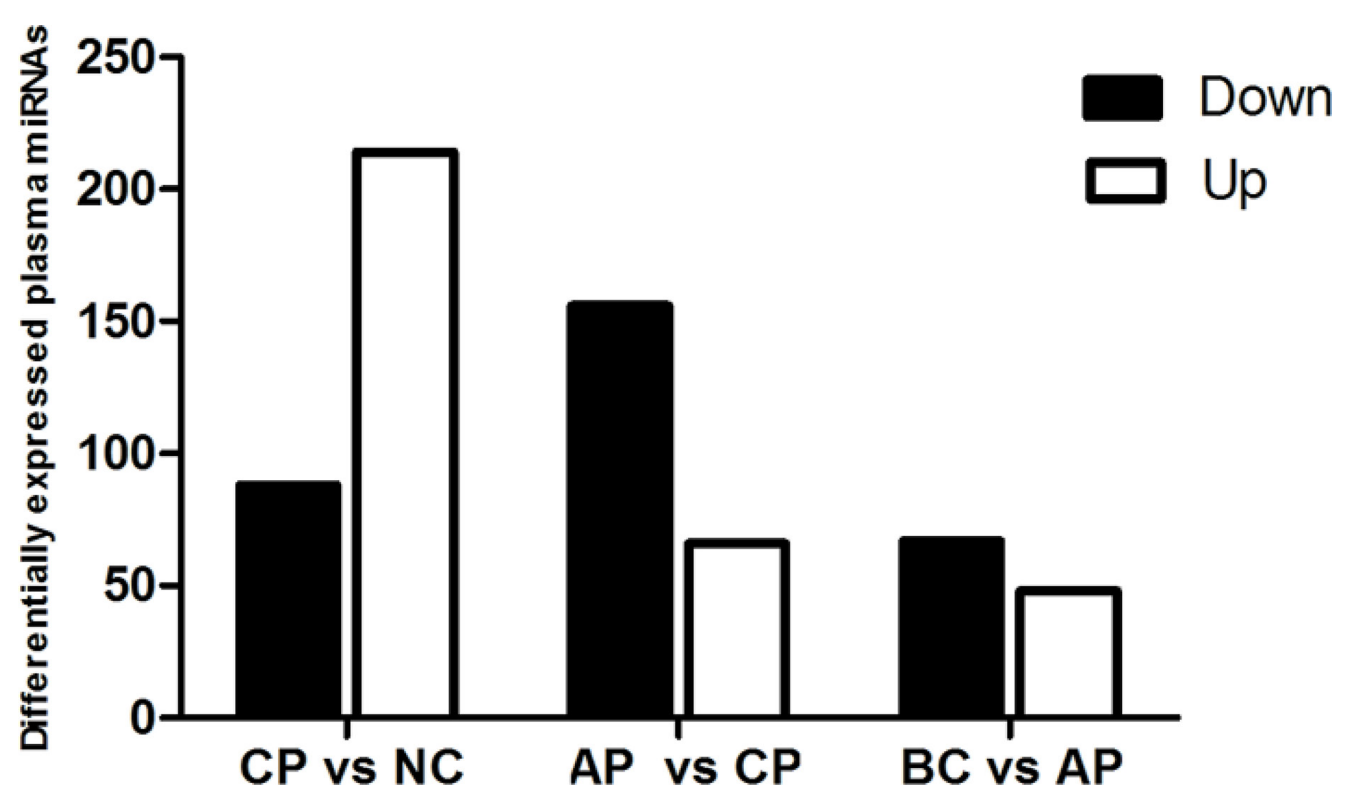

Figure 2: Representation of differential miRNA expression at different phases of CML. Differetially expressed miRNAs were analyzed between phases. The threshold value for up- and down-regulated genes was a fold change $\geq 2.0$.

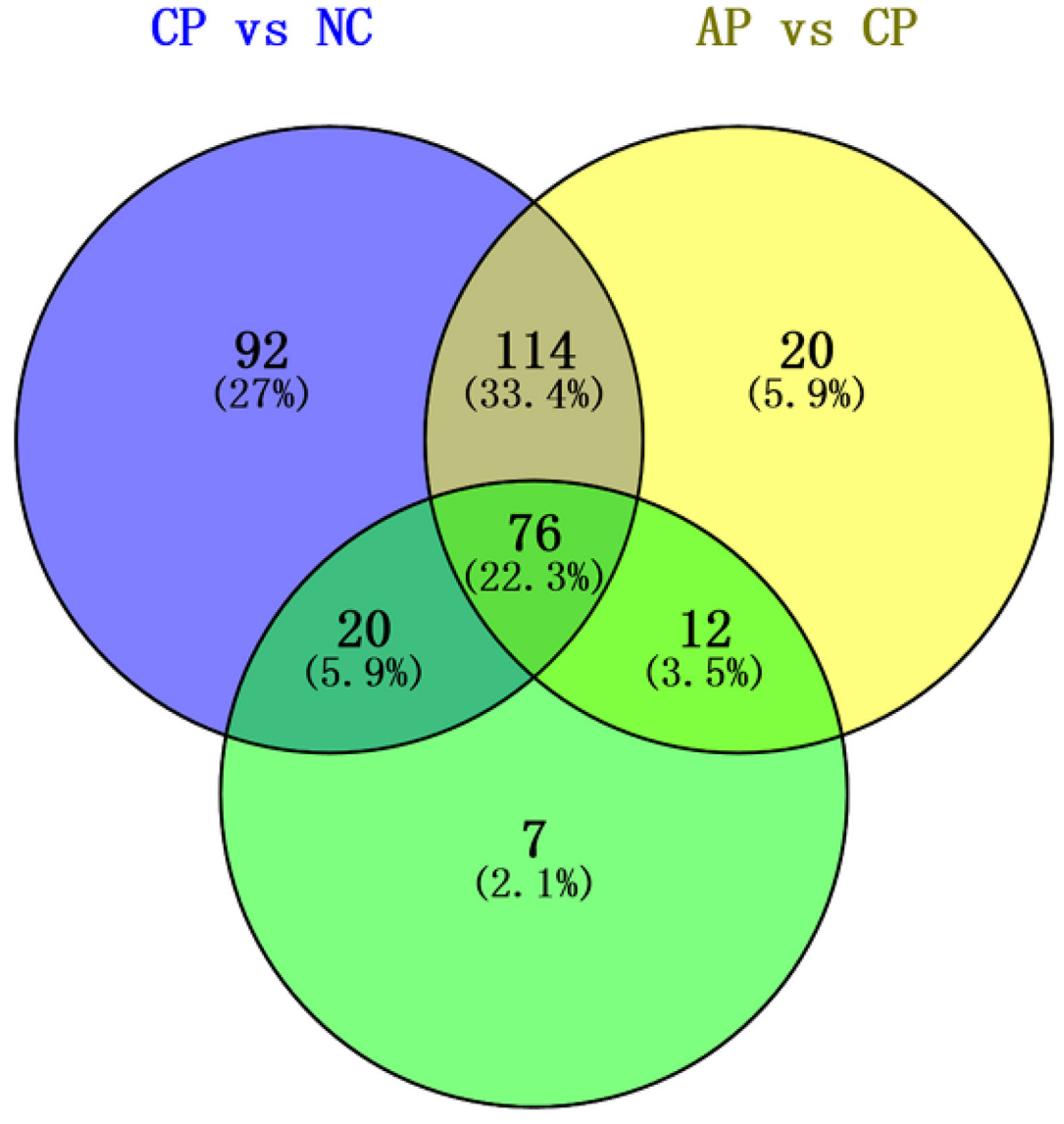

$\mathrm{BC}$ vs AP

Figure 3: Venn analysis of differential miRNA expression between different phases of CML. Differetially expressed miRNAs were analyzed among multiple comparison. The threshold value for up- and down-regulated genes was a fold change $\geq 2.0$. 
significantly increased and restored to a normal range. There was no significant change between the healthy controls and MMR patients.

To estimate the clinical value of the plasma miR451a expression across the progression of CML, we used receiver operating characteristic (ROC) curves (Figure 8) to distinguish between different groups. Figure 8A shows that plasma miR-451a yielded an AUC (the area under the ROC curve) of $0.732(p=0.002)(95 \%$ confidence interval [CI] $0.603-0.862$ ) with $65.0 \%$ sensitivity and $67.2 \%$ specificity for discriminating CML patients from healthy controls at a cut-off value of 5.08. miR-451a expression significantly decreased in AP and BC patients. Figure 8C shows that the AUC was $0.844(p=0.000)(95 \% \mathrm{CI}$ 0.724-0.965), suggesting that the plasma miR-451a level is down-regulated distinctly in AP/BC patients compared with healthy samples. Furthermore, Figure $8 \mathrm{D}$ shows that the AUC was 0.724 for miR-451a ( $p=0.007)(95 \% \mathrm{CI}$ $0.588-0.895)$ with $62.5 \%$ sensitivity and $72.2 \%$ specificity for discriminating $\mathrm{CP}$ and $\mathrm{AP} / \mathrm{BC}$ at a cut-off value of 1.74 .

\section{DISCUSSION}

It was recently reported that plasma and serum samples contained sufficiently stable miRNA signatures and that circulating miRNAs can be identified and measured accurately. Compared to conventional diagnostic parameters, the circulating miRNA profile is appropriate for diagnosing diseases as well as estimating their progression and therapeutic outcomes with higher specificity and sensitivity. The role of the Bcr-abl oncogene has been demonstrated in the pathogenesis of CML; however, the mechanisms at the advanced phases of CML remain unclear. In addition, more patients are resistant to tyrosine-kinase inhibitor (TKI) in the later stages, suggesting that other genetic abnormalities may be involved. We focused on the relationship between plasma miRNA profiles and disease progression. The circulating miRNA profiles may provide a better understanding of the in vivo intercellular communication in CML patients.
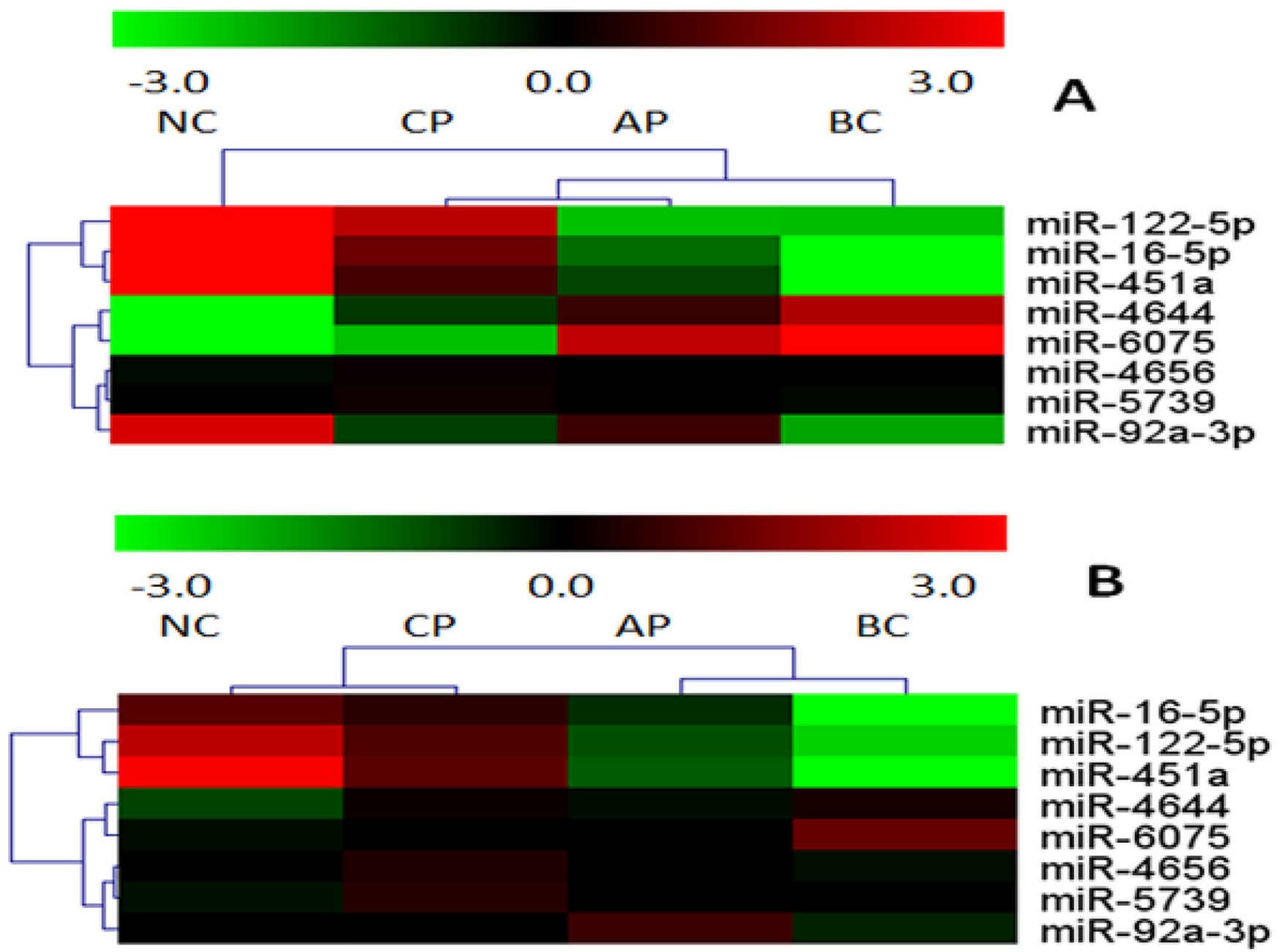

Figure 4: Hierarchical clustering of plasma miRNA expression data. (A) microarray-pools, (B) real-time qPCR-sample pools. Eight candidate miRNAs were chosen to evaluate the microarray data and pooling precision by qRT-PCR, including four down-regulated miRNAs (miR-122-5p, miR-16-5p, miR-451a and miR-92a-3p), two up-regulated miRNAs (miR-4644 and miR-6075), and two stably expressed miRNAs (miR-4656 and miR-5739). 
Table 1: Characteristics of study subjects in the pools

\begin{tabular}{lllccccc}
\hline Pools & $\begin{array}{c}\text { No of patients } \\
\text { (gender) }\end{array}$ & Age & $\begin{array}{c}\text { WBC count } \\
(\times \mathbf{1 0} / \mathbf{L})\end{array}$ & $\begin{array}{c}\text { Thrombocyte } \\
\text { count }(\times \mathbf{1 0} / \mathbf{L})\end{array}$ & $\begin{array}{c}\text { Hemoglobin } \\
(\mathbf{g} / \mathbf{L})\end{array}$ & $\begin{array}{c}\text { Bcr-abl } \\
\text { (Positive/ } \\
\text { Negative) }\end{array}$ & $\begin{array}{c}\text { Therapy } \\
\text { months }\end{array}$ \\
\hline \multirow{4}{*}{ NC } & 1 Male & 55 & 9.28 & 196 & 146 & Negative & 0 \\
& 2 Female & 41 & 9.84 & 266 & 134 & Negative & 0 \\
& 3 Female & 44 & 5.41 & 165 & 118 & Negative & 0 \\
& 4 Male & 25 & 8.88 & 278 & 149 & Negative & 0 \\
& 5 Male & 58 & 130.82 & 550 & 82 & Positive & 0 \\
CP & 6 Male & 49 & 37.65 & 11 & 77 & Positive & 0 \\
& 7 Female & 60 & 183.24 & 447 & 74 & Positive & 0 \\
& 8 Male & 66 & 303.88 & 130 & 70 & Positive & 0 \\
& 9 Female & 55 & 28.88 & 264 & 70 & Positive & 8.1 \\
AP & 10 Male & 50 & 13.02 & 555 & 109 & Positive & 32.8 \\
& 11 Female & 45 & 1.66 & 3 & 23 & Positive & 55.3 \\
& 12 Female & 54 & 378.59 & 184 & 57 & Positive & 39.4 \\
& 13 Female & 35 & 1.94 & 8 & 38 & Positive & 60.5 \\
BC & 14 Male & 21 & 12.46 & 150 & 129 & Positive & 37.8 \\
& 15 Female & 29 & 28.88 & 19 & 98 & Positive & 10.2 \\
& 16 Female & 59 & 16.18 & 297 & 53 & Positive & 45.9 \\
\hline
\end{tabular}

NC: normal control; CP: chronic phase; AP accelerated phase; BC: blast crisis

Previous study has confirmed a relationship between clinical outcome of patients with acute myeloid leukemia and circulating miRNAs [19]. Moreover, The exosomal miR-140- $3 p$ is significantly elevated in CML patients with musculoskeletal pain compared to those without pain and healthy individuals [20].

MicroRNA-seq is an effective technique in miRNA identification, and in particular, microRNA-seq also has the notable advantage of distinguishing among unknown RNA species that would not be detected by microarray [21, 22]. However, it is less frequently used due to the increased starting material requirements and costly expense. In contrast, microarray can be performed with less amount of material [11,23]. As miRNA abundance in plasma is very low, and the total amount of miRNA extracted by using miReasy Serum/Plasma kit is very little, microarray is still widely used in the detection of plasma miRNA [24-26].

We applied the functional annotation tool, DAVID, to reveal the biological functions of putative targets with conserved sites and high $P_{\mathrm{CT}}$ values for the strongly dysregulated miRNAs between different phases. Using the KEGG pathways to analyze targets of dysregulated miRNAs across the progression of CML, we have found that many dysregulated potential target molecules were involved in several biological pathways including metabolic and cancer pathways, the MAPK signaling pathway, endocytosis, cytokine-cytokine receptor interaction and focal adhesion. Recent studies have confirmed that pathogenesis and progression of CML were closely related to the MAPK signaling pathway [27, 28], endocytosis $[29,30]$ and focal adhesion [31, 32].

To better understand this leukemogenesis, we investigated the dynamics of miRNA levels during the progression of CML. We selected four patterns to explore. The dynamically increased miRNAs participated in a series of biological processes including the MAPK cascade, homophilic cell adhesion via plasma membrane adhesion molecules, synaptic transmission and axon guidance. They were closely related to the MAPK signaling pathway, cAMP signaling pathway, focal adhesion and endocytosis. Moreover, dynamically reduced miRNAs were involved mainly in the PI3K-Akt signaling pathway, signaling pathways regulating the pluripotency of stem cells, the MAPK signaling pathway, focal adhesion and cancer pathways.

This study used real-time qPCR to validate the down-regulation of miR-451a in four sample pools and a larger cohort of individual samples. miR-451a decreased dramatically during the progression, which is related to CML pathogenesis and may reflect a transformation from the chronic to accelerated phases and blast crisis. Lopotová $\mathrm{T}$ et al. found that miR-451a was down-regulated in CML patients compared to healthy controls, and a complex relationship between miR-451a and BCR-ABL was revealed in CML [17]. Down-regulated miR-451a was observed in imatinib-resistant CML cases in which miR-451a and MYC formed a regulatory loop that may be a potential therapeutic target for CML [15]. Furthermore, it was confirmed that 
miR-451a was significantly down-regulated in IM-resistant compared to IM-responsive patients [16]. The expression level of miR-451a in CML plasma was consistent with that in leukemia cells. These results suggest that miR451a plays an important regulatory role in pathogenesis and drug-resistance in the progression of CML. Other studies demonstrated that the down-regulated expression of miR-451a was closely related to the pathogenesis of various hematological malignancies involved in multiple myeloma [33, 34], acute myeloid leukemia [35, 36], acute lymphoblastic leukemia [37, 38], myelodysplastic syndrome [39] and polycythemia vera [40]. In addition, miR-451a was required for erythroid homeostasis [41]. Svasti S et al. demonstrated that early erythroid progenitors in $\beta$-thalassemia have possessed a dysregulated miRNA-451a expression program [42].

To further elucidate the possible role of miR451a across CML progression, we detected a larger

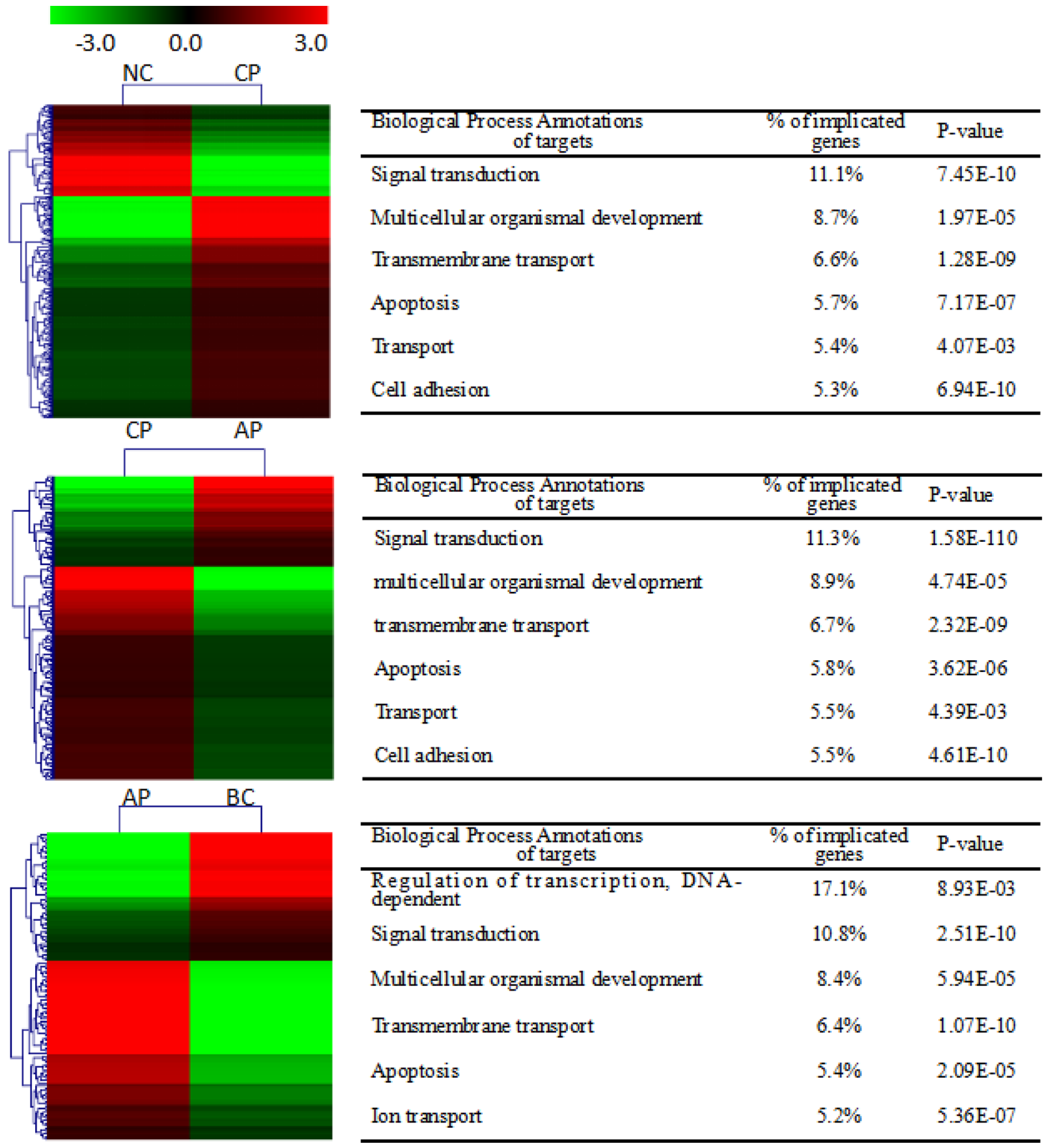

Figure 5: Clusters of differentially expressed miRNA and GO analysis of their targets in CML among the different phases. The heatmap showed all differentially expressed plasma miRNAs between different phases among disease progression. Gene Ontology (GO) analysis were applied to annotate the biological functions of their predicted targets. 
cohort of individual samples including CP, AP and BC in comparison to healthy individuals. Plasma expression levels of miR-451a were significantly low, and expression gradually decreased from $\mathrm{CP}$ to $\mathrm{AP}$ and $\mathrm{BC}$. The downregulation of miR-451a may be associated with the advanced disease stage of CML. However, when CML patients achieved MMR, miR-451a expression increased and was restored to normal range. This suggests that miR451a can function as a potential biological biomarker for diagnosis and evaluation of the prognosis of CML. Our observations of CML patients were consistent with those in other cancers. Redova $\mathrm{M}$ et al. confirmed that miR-451a level was decreased in the serum of patients with renal cell carcinoma (RCC), and a combination of miR-451a with miR-378 can distinguish RCC serum with $81 \%$ sensitivity,
$83 \%$ specificity and an AUC $=0.86$ [43]. Serum miR451a levels were significantly decreased in breast cancer, and higher serum levels were associated with improved clinical and pathological responses and disease-free survival $[44,45]$.

In conclusion, it is not clear how $\mathrm{CP}$ patients progress into the advanced stages including $\mathrm{BP}$ and $\mathrm{BC}$. Our data may provide insight into the disease pathobiology, allowing an understanding of the mechanisms of the advanced disease, and may provide insight for identifying potential biomarkers and therapeutic targets in CML. There are fundamental differences in plasma miRNA expression levels among the different phases of CML. We applied Gene Ontology and KEGG pathway analysis to annotate the biological functions of dysregulated miRNAs.
Group 1

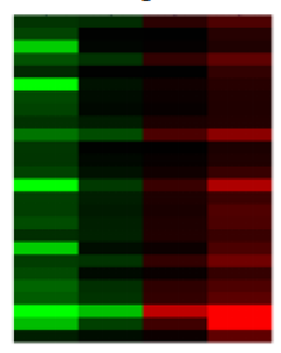

Group 2

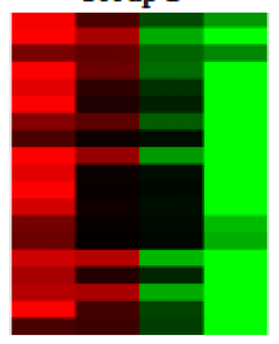

Group 3
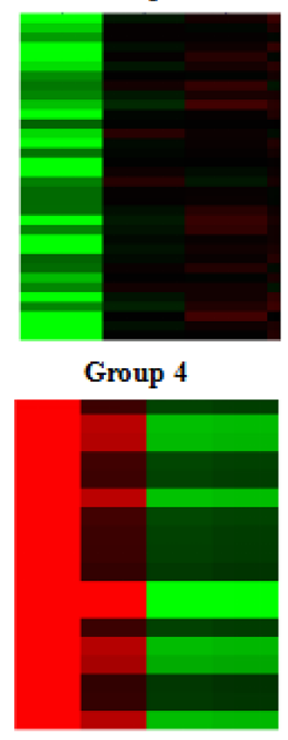
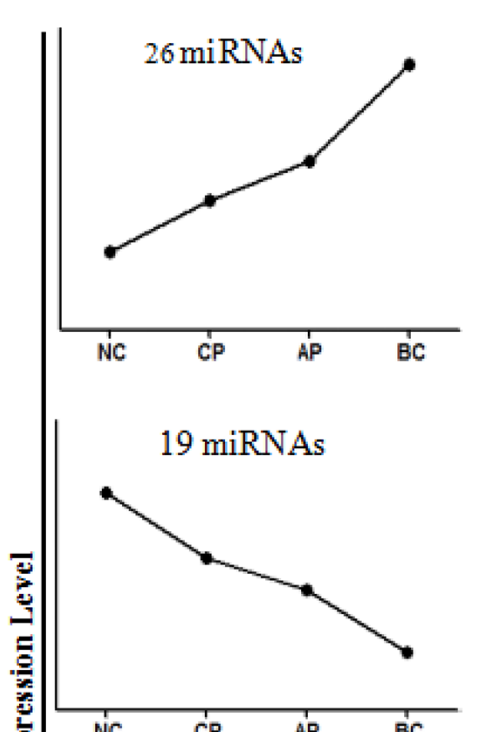

NC $\quad \dot{C P} \quad \dot{A P} \quad \dot{B C}$

\section{4 miRNAs}
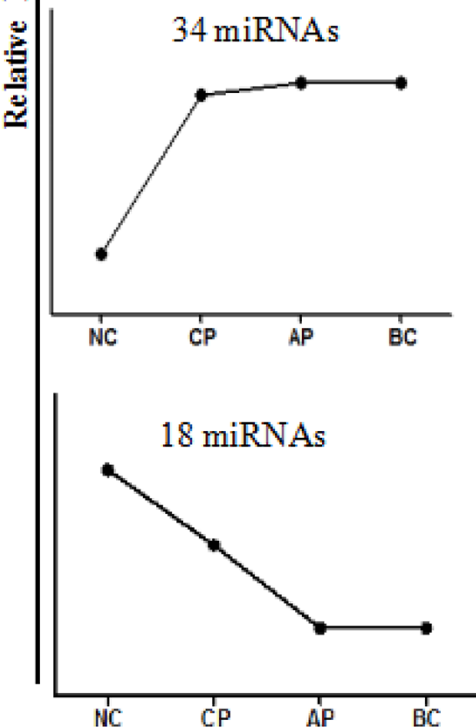

\begin{tabular}{|c|c|}
\hline Bidogical Process Annotations of targets & P-value \\
\hline $\begin{array}{l}\text { Homophilic cell adhesion via plasma Membrane adhesion } \\
\text { molecules }\end{array}$ & $8 \mathrm{E}-17$ \\
\hline Nervous system development & $1.2 \mathrm{E}-12$ \\
\hline Axon guidance & $1.7 \mathrm{E}-09$ \\
\hline Synaptic transmissicn & $6.2 \mathrm{E}-06$ \\
\hline MAPK cascade & $5.4 \mathrm{E}-05$ \\
\hline Histone H4-K12 acetylation & $6.5 \mathrm{E}-05$ \\
\hline Bidogical Process Annotations of targets & P-value \\
\hline Positive regulation of transcription, DNA-templated & $6.2 \mathbb{E}-09$ \\
\hline $\begin{array}{l}\text { Positive regulation of transcription from RNA polymerase } \\
\text { II promoter }\end{array}$ & 3.2E-08 \\
\hline Nervous system development & 4.2E-08 \\
\hline Transcription from RNA polymerase II promoter & $3 . \mathrm{E}-07$ \\
\hline Negative regulation of cell migration & 4.1E-05 \\
\hline Axon guidance & $6.3 \mathrm{E}-05$ \\
\hline Biological Process Annotations of targets & P-value \\
\hline $\begin{array}{l}\text { Positive regulation of transcription from RNA polymerase II } \\
\text { promoter }\end{array}$ & $1.9 \mathrm{E}-09$ \\
\hline Transcription from RNA polymerase II promoter & 1.7E-08 \\
\hline $\begin{array}{l}\text { Negative regulation of transcription from RNA polymerase } \\
\text { II promoter }\end{array}$ & $3.4 \mathrm{E}-07$ \\
\hline Transcription, DNA-templated & 2.2E-06 \\
\hline Axon guidance & $4.9 \mathrm{E}-06$ \\
\hline Brain devel opment & $6.1 \mathrm{E}-05$ \\
\hline Biological Process Annotations of targets & P-value \\
\hline Positive regulation of transcription, DNA-templated & 4.3E-10 \\
\hline Axon guidance & $5.1 \mathrm{E}-08$ \\
\hline Nervous system development & $3.1 \mathrm{E}-07$ \\
\hline $\begin{array}{l}\text { Positive regulation of transcription from RNA polymerase II } \\
\text { promoter }\end{array}$ & 3.3E-07 \\
\hline Protein phosphorylation & $5.1 \mathrm{E}-07$ \\
\hline Activation of protein kinase activity & 1.3E-06 \\
\hline
\end{tabular}

Figure 6: Clusters of miRNA expression across the progression of CML. The heatmap showed the dynamics of miRNA expression levels during the progression of CML. Gene Ontology (GO) analysis were applied to annotate the biological functions of their predicted targets. 
This may provide novel insight into the differences between the different phases of the disease progression.

\section{MATERIALS AND METHODS}

\section{Ethics statement}

The study was approved by the institutional ethics committee of the First Affiliated Hospital of South China University and Xiangya Hospital of Central South University. All patients signed an informed consent form approved. We have been obtained consent to publish individual participant's data by the participant or legal parent.

\section{Patients and sample preparation}

Plasma samples from CML patients and healthy controls were collected in EDTA-K2 tubes and centrifuged at 3,000 rpm for $15 \mathrm{mins}$ at room temperature. The supernatants were collected, centrifuged at 15,000 rpm for 30 mins at $4^{\circ} \mathrm{C}$ and stored at $-80^{\circ} \mathrm{C}$ until RNA extraction.

To optimize this detection step and identify the dysregulated miRNAs, plasma sample pools were applied for the microarray analysis to reduce individual variability and find common features of the CML at different phases of disease progression. A pooling strategy was conducted according to previously described recommendations
[46]. Moreover, the pooling strategy to detect circulating miRNA profiles have been applied in a series of patients with multiple myeloma [46], acute myeloid leukemia [19] and breast cancer [47]. Each patient sample was equally important because it contributed to the same volume of plasma. Therefore, this procedure reduced variation between individuals and enriched the miRNAs most likely to change between CML patients in different phases as well as the control group. Twelve patient plasma samples and four healthy plasma samples obtained from peripheral blood (Table 1) were used to prepare pools representing the different CML phases for the microarrays, and they were divided according to diagnostic criteria. Newly diagnosed chronic-phase CML patients $(n=4)$, accelerated- phase CML patients $(n=4)$, blast crisis CML patients $(n=4)$ and healthy donors $(n=4)$ were used to create the sample pools.

A total of 98 plasma samples were used to validate the miR-451a expression involved in CP $(n=40)$, AP together with $\mathrm{BC}(\mathrm{AP} / \mathrm{BC})(n=18), \mathrm{MMR}(n=20)$ and healthy donors $(n=20)$. The Bcr-abl gene was detected by real-time qPCR or fluorescence in situ hybridization (FISH).

\section{RNA extraction}

Total plasma miRNA was extracted from $200 \mu \mathrm{l}$ samples using miReasy Serum/Plasma kit (Qiagen, Germany) according to the manufacturer's protocol. Synthetic cel-miR-39 was added to each sample as an

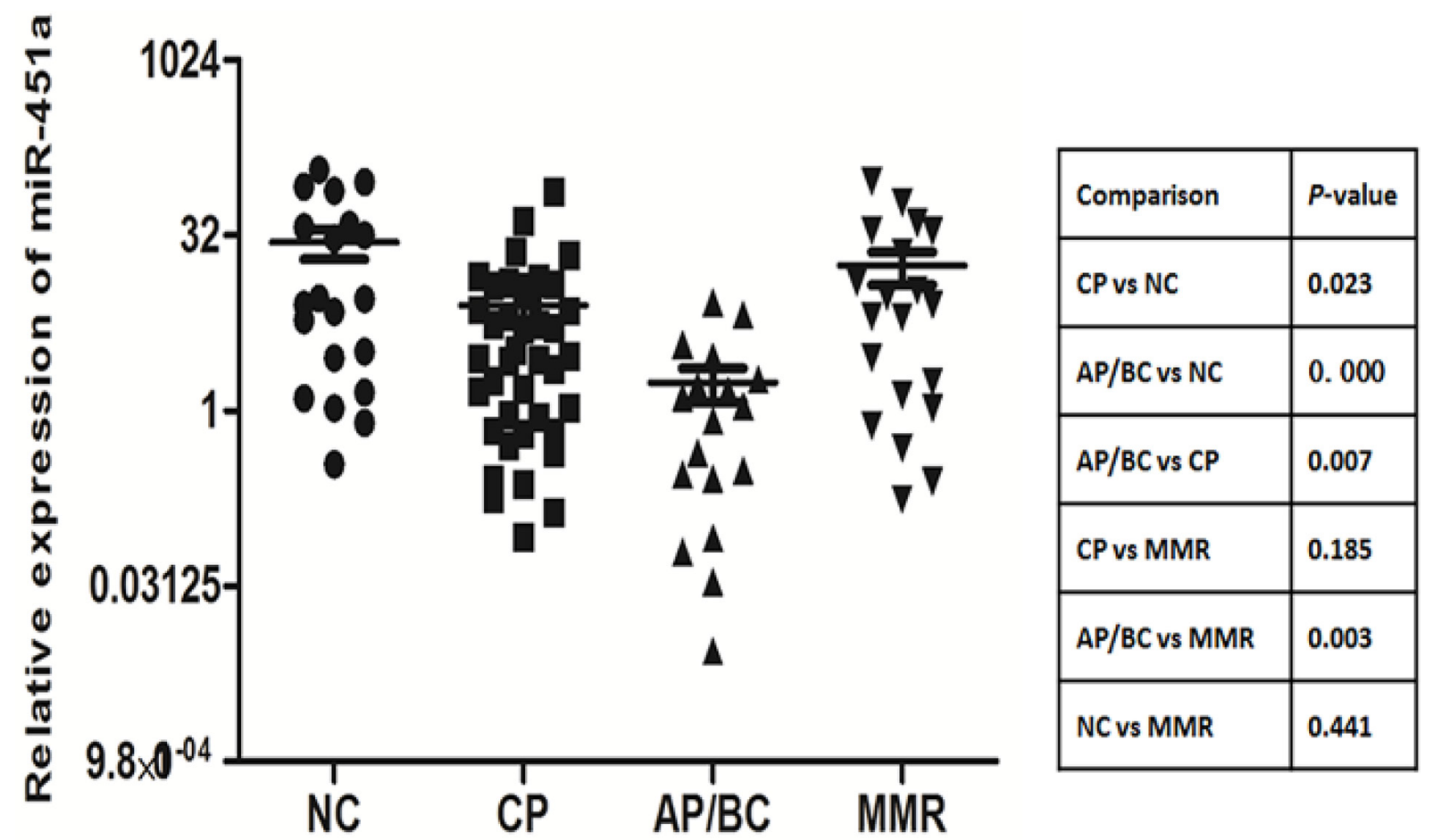

Figure 7: Expression of plasma miRNA-451a across the disease progression of CML. Expression of plasma miR-451a was detected by qRT-PCR across the disease progression of CML. The Mann-Whitney $U$ test was employed to determine the difference in a larger cohort of samples between different groups, and $p$ values $<0.05$ were considered significant. 
internal control for evaluation of successful extraction. RNAs were eluted twice with $14 \mu \mathrm{L}$ of RNase-free water, and the RNA concentration was quantified by a NanoDrop ND-1000 spectrometer (Thermo Scientific, USA).

\section{Plasma miRNA microarray analysis and expression profiling}

To reveal the expression profile of the plasma miRNAs at the different phases of CML, we performed miRNA expression profiling of the pooled samples including $\mathrm{CP}$, AP and $\mathrm{BC}$, as well as healthy control samples. Agilent Human miRNA $(8 \times 60 \mathrm{~K}$, Design ID:046064) was used per the manufacturer's instructions. The miRNA profiling microarrays were conducted by the China Shanghai OE Technology Co., Ltd. Feature Extraction software (version 10.7.1.1, Agilent Technologies) was used to analyze array images for the
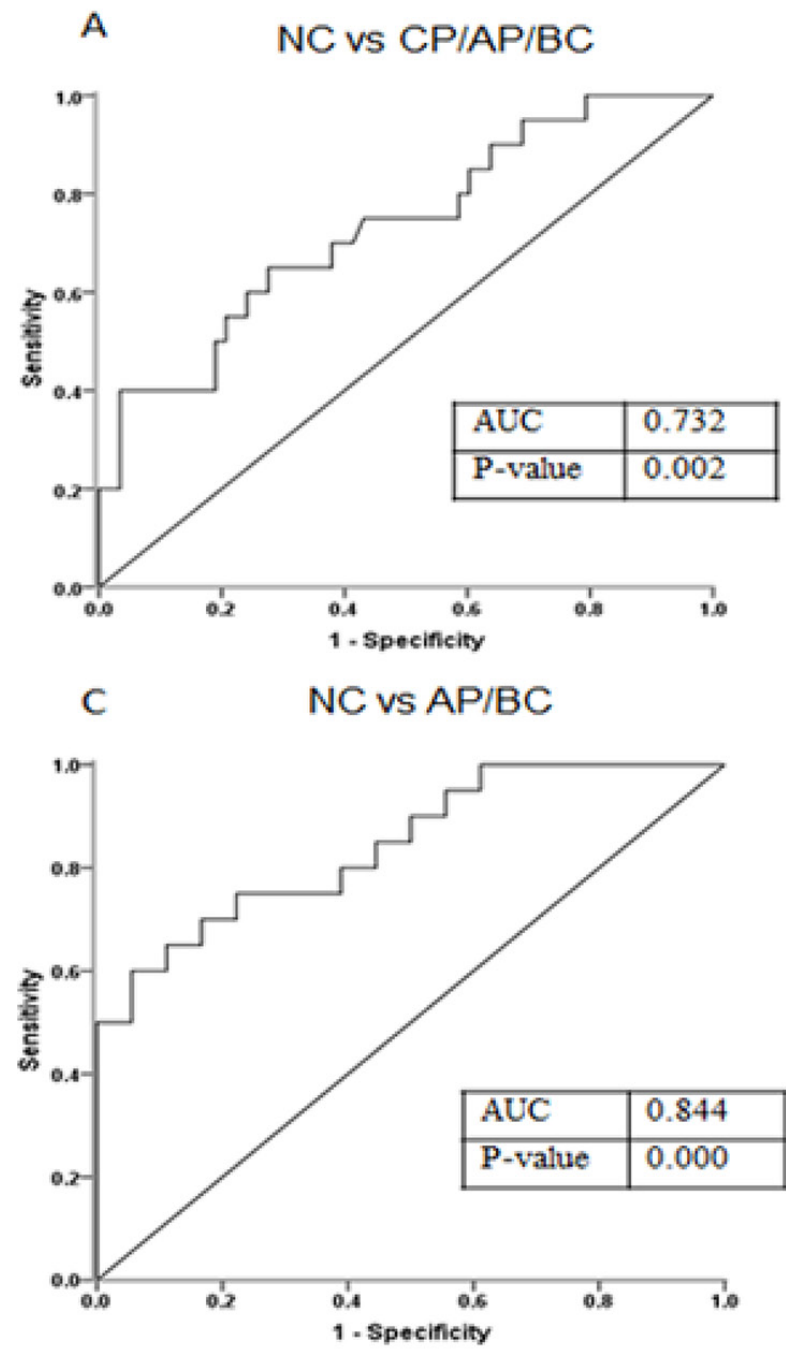

raw data. Genespring software (version 12.5, Agilent Technologies) was used to finish the basic analysis of the raw data. The raw data was normalized with the quantile algorithm, and differentially expressed miRNAs were then identified through fold change. The threshold value for upand down-regulated genes was a fold change $\geq 2.0$.

\section{qRT-PCR for validation of independent individual miRNAs}

Candidate miRNAs were detected by qRT-PCR with miRNA-specific stem-loop primers. Quantitative PCR for miRNA was preformed using the Mastercycler EP Gradient (Eppendorf, Germany). The reaction was initiated under the following conditions: $95^{\circ} \mathrm{C}$ for $2 \mathrm{~min}$, 50 cycles of $95^{\circ} \mathrm{C}$ for $15 \mathrm{~s}$ and $60^{\circ} \mathrm{C}$ for $60 \mathrm{~s}$. Each assay was conducted in duplicate. Data points that generated duplicate $\mathrm{Ct}$ values with over one cycle variance were
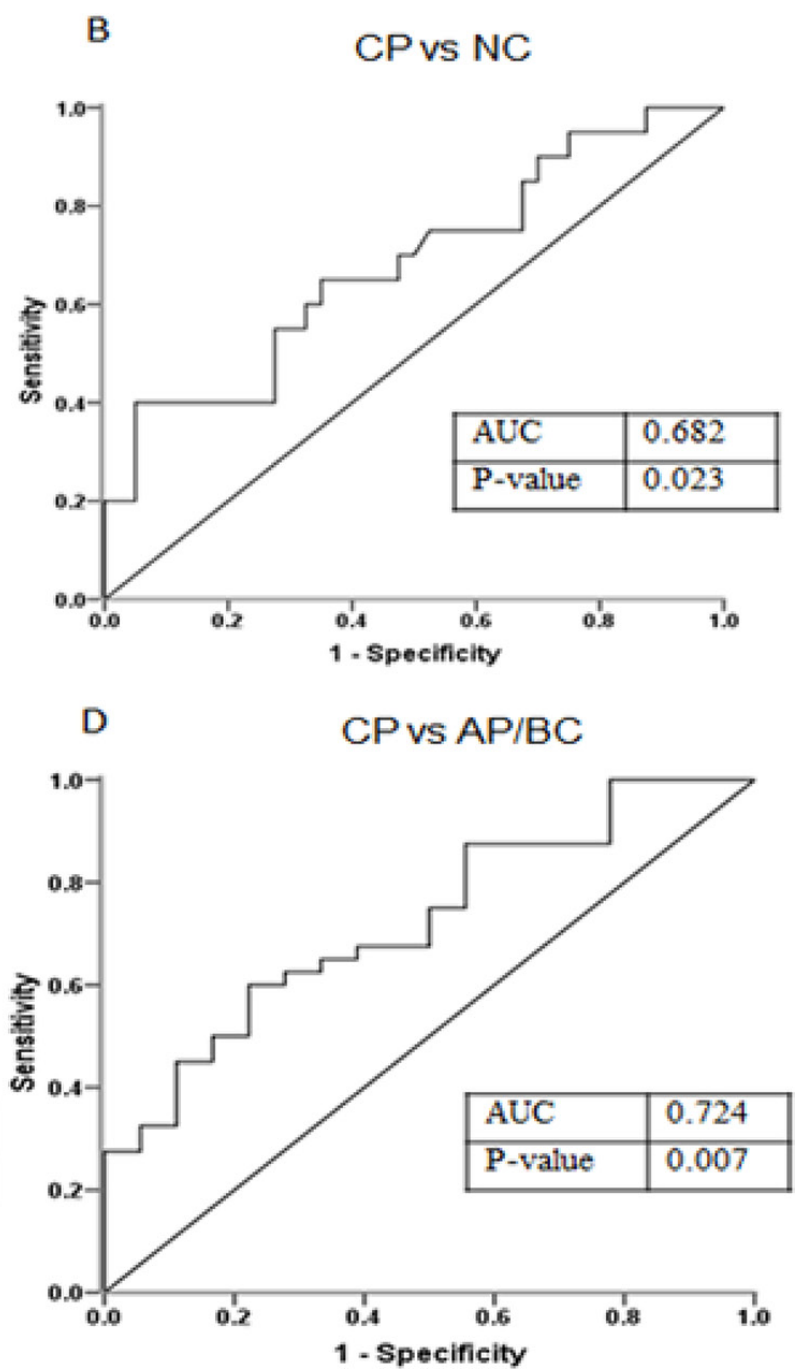

Figure 8: ROC curve of miR-451a to distinguish between different groups in progression of CML. (A) miR-451a can clearly distinguish between $\mathrm{NC}$ and $\mathrm{CML}$ groups $(\mathrm{AUC}=0.732, p=0.002)$. (B) and $(\mathbf{C})$ miR-451a can distinguish between $\mathrm{NC}$ and $\mathrm{CP}$ groups and $\mathrm{AP} / \mathrm{BC}$ groups; $(\mathrm{AUC}=0.682, p=0.023)$ and $(\mathrm{AUC}=0.844, p<0.001)$, respectively. $(\mathrm{D})$ miR-451a can clearly distinguish between $\mathrm{CP}$ and $\mathrm{AP} / \mathrm{BC}$ groups $(\mathrm{AUC}=0.724, p=0.007)$. 
excluded from analysis. The spike control (cel-miR-39) was used as an invariant control for the plasma miRNA. The $\Delta \mathrm{Ct}$ method was used for analysis $[\Delta \mathrm{Ct}=$ average $\mathrm{Ct}$ (miRNA of target)-average $\mathrm{Ct}$ (cel-39]. The relative expression of the miRNAs was calculated by the equation $2^{-\Delta \mathrm{Ct}}[48]$.

\section{Statistical analysis}

Data were statistically analyzed using SPSS version 16.0 (SPSS, Inc, Chicago, IL, USA). The Mann-Whitney $U$ test was employed to determine the difference in a larger cohort of clinical samples between different groups. We constructed the receiver operating characteristic (ROC) curve and calculated the area under the ROC. $p$ values $<0.05$ were considered significant for all analyses.

\section{Bioinformatics analysis}

The TargetScan Human release 7.1 (http://www. targetscan.org) was applied to predict putative targets of the miRNA [18]. The predicted targets containing highly conserved binding sites were further studied according to the $P_{\mathrm{CT}}$ values from the TargetScan release 7.1 [49]. The putative targets were selected according to the $\mathrm{P}_{\mathrm{CT}} \geq$ 0.1 and 0.5 for the miRNAs with low and high numbers of targets in the database, respectively. Gene Ontology (GO) analysis and the Kyoto Encyclopedia of Genes and Genomes (KEGG) pathway were applied to annotate the biological functions of the predicted targets [50].

\section{Abbreviations}

CML: chronic myeloid leukemia; CP: chronic phase; AP: accelerated phase; BC: blast crisis; DAVID: Database for Annotation, Visualization and Integrated Discovery; ROC: receiver operating characteristic; GO: Gene Ontology; KEGG: Kyoto Encyclopedia of Genes and Genomes; AUC: Area under ROC curve; CI: confidence interval.

\section{Author contributions}

$\mathrm{JL}$ and $\mathrm{JZ}$ designed the research study. JZ, XZ, XH, WL and YJ collected patients' samples and performed the qRT-PCR. JZ analyzed the data. JZ and JL wrote the manuscript. All authors read and approved the final manuscript.

\section{ACKNOWLEDGMENTS AND FUNDING}

This work was supported by the National Natural Science Foundation of China (81301774 and 81470362) and Natural Science Foundation of Hunan Province (2016JJ2108).

\section{CONFLICTS OF INTEREST}

The authors declare that they have no competing interests.

\section{REFERENCES}

1. Rowley JD. Letter: A new consistent chromosomal abnormality in chronic myelogenous leukaemia identified by quinacrine fluorescence and Giemsa staining. Nature. 1973; 243:290-293.

2. Hughes A, Yong ASM. Immune Effector Recovery in Chronic Myeloid Leukemia and Treatment-Free Remission. Front Immunol. 2017; 8:469.

3. Crisan AM, Coriu D, Arion C, Colita A, Jardan C. The impact of additional cytogenetic abnormalities at diagnosis and during therapy with tyrosine kinase inhibitors in Chronic Myeloid Leukaemia. J Med Life. 2015; 8:502-508.

4. Salati S, Salvestrini V, Carretta C, Genovese E, Rontauroli S, Zini R, Rossi C, Ruberti S, Bianchi E, Barbieri G, Curti A, Castagnetti F, Gugliotta G, et al. Deregulated expression of miR-29a-3p, miR-494-3p and miR-660-5p affects sensitivity to tyrosine kinase inhibitors in CML leukemic stem cells. Oncotarget. 2017; 8:49451-49469. http://doi. org/10.18632/oncotarget.17706.

5. Vasilatou D, Papageorgiou S, Pappa V, Papageorgiou E, Dervenoulas J. The role of microRNAs in normal and malignant hematopoiesis. Eur J Haematol. 2010; 84:1-16.

6. Fang ZH, Wang SL, Zhao JT, Lin ZJ, Chen LY, Su R, Xie ST, Carter BZ, Xu B. miR-150 exerts antileukemia activity in vitro and in vivo through regulating genes in multiple pathways. Cell Death Dis. 2016; 7:e2371.

7. de Candia P, Torri A, Pagani M, Abrignani S. Serum microRNAs as Biomarkers of Human Lymphocyte Activation in Health and Disease. Front Immunol. 2014; 5:43.

8. Liu Y, Luo F, Wang B, Li H, Xu Y, Liu X, Shi L, Lu X, $\mathrm{Xu}$ W, Lu L, Qin Y, Xiang Q, Liu Q. STAT3-regulated exosomal miR-21 promotes angiogenesis and is involved in neoplastic processes of transformed human bronchial epithelial cells. Cancer Lett. 2016; 370:125-135.

9. Bertoli G, Cava C, Castiglioni I. MicroRNAs: New Biomarkers for Diagnosis, Prognosis, Therapy Prediction and Therapeutic Tools for Breast Cancer. Theranostics. 2015; 5:1122-1143.

10. Casadei L, Calore F, Creighton CJ, Guescini M, Batte K, Iwenofu OH, Zewdu A, Braggio D, Bill KL, Fadda P, Lovat F, Lopez G, Gasparini P, et al. Exosome-derived miR-25-3p and miR-92a-3p stimulate liposarcoma progression. Cancer Res. 2017; 77:3846-3856.

11. Zhang J, Xiao X, Liu J. The role of circulating miRNAs in multiple myeloma. Sci China Life Sci. 2015; 58:1262-1269.

12. Taverna S, Amodeo V, Saieva L, Russo A, Giallombardo M, De Leo G, Alessandro R. Exosomal shuttling of miR-126 in 
endothelial cells modulates adhesive and migratory abilities of chronic myelogenous leukemia cells. Mol Cancer. 2014; 13:169.

13. Ohyashiki K, Umezu T, Katagiri S, Kobayashi C, Azuma K, Tauchi T, Okabe S, Fukuoka Y, Ohyashiki JH. Downregulation of Plasma miR-215 in Chronic Myeloid Leukemia Patients with Successful Discontinuation of Imatinib. Int J Mol Sci. 2016; 17:570.

14. Taverna S, Giallombardo M, Pucci M, Flugy A, Manno M, Raccosta S, Rolfo C, De Leo G, Alessandro R. Curcumin inhibits in vitro and in vivo chronic myelogenous leukemia cells growth: a possible role for exosomal disposal of miR-21. Oncotarget. 2015; 6:21918-21933. http://doi. org/10.18632/oncotarget.4204.

15. Soltani I, Douzi K, Gharbi H, Benhassine I, Teber M, Amouri H, Ben Hadj Othman H, Farrah A, Ben Lakhel R, Abbes S, Menif S. Downregulation of miR-451 in Tunisian chronic myeloid leukemia patients: potential implication in imatinib resistance. Hematology. 2017; 22:201-207.

16. Jurkovicova D, Lukackova R, Magyerkova M, Kulcsar L, Krivjanska M, Krivjansky V, Chovanec M. microRNA expression profiling as supportive diagnostic and therapy prediction tool in chronic myeloid leukemia. Neoplasma. 2015; 62:949-958.

17. Lopotova T, Zackova M, Klamova H, Moravcova J. MicroRNA-451 in chronic myeloid leukemia: miR-451BCR-ABL regulatory loop? Leuk Res. 2011; 35:974-977.

18. Machova Polakova K, Lopotova T, Klamova H, Burda P, Trneny M, Stopka T, Moravcova J. Expression patterns of microRNAs associated with CML phases and their disease related targets. Mol Cancer. 2011; 10:41.

19. Han GT, Sun ZL. Up-regulation of serum miR-4262 predicts clinical outcome of patients with acute myeloid leukemia. Eur Rev Med Pharmacol Sci. 2017; 21:2172-2176.

20. Asano M, Umezu T, Katagiri S, Kobayashi C, Tauchi T, Gotoh M, Ando K, Okabe S, Ohyashiki JH, Ohyashiki K. Up-regulated exosomal miRNA-140-3p in CML patients with musculoskeletal pain associated with discontinuation of tyrosine kinase inhibitors. Int J Hematol. 2017; 105:419-422.

21. Rai G, Rai R, Saeidian AH, Rai M. Microarray to deep sequencing: transcriptome and miRNA profiling to elucidate molecular pathways in systemic lupus erythematosus. Immunol Res. 2016; 64:14-24.

22. Selth LA, Tilley WD, Butler LM. Circulating microRNAs: macro-utility as markers of prostate cancer? Endocr Relat Cancer. 2012; 19:R99-R113.

23. Gao L, Jiang F. MicroRNA (miRNA) Profiling. Methods Mol Biol. 2016; 1381:151-161.

24. Yan S, Han B, Gao S, Wang X, Wang Z, Wang F, Zhang J, Xu D, Sun B. Exosome-encapsulated microRNAs as circulating biomarkers for colorectal cancer. Oncotarget. 2017; 8:6014960158. http://doi.org/10.18632/oncotarget.18557.

25. Akiyoshi S, Fukagawa T, Ueo H, Ishibashi M, Takahashi Y, Fabbri M, Sasako M, Maehara Y, Mimori K, Mori M.
Clinical significance of miR-144-ZFX axis in disseminated tumour cells in bone marrow in gastric cancer cases. Br J Cancer. 2012; 107:1345-1353.

26. Ren C, Liu Q, Wei Q, Cai W, He M, Du Y, Xu D, Wu Y, Yu J. Circulating miRNAs as Potential Biomarkers of AgeRelated Macular Degeneration. Cell Physiol Biochem. 2017; 41:1413-1423.

27. Chakraborty C, Sharma AR, Patra BC, Bhattacharya M, Sharma G, Lee SS. MicroRNAs mediated regulation of MAPK signaling pathways in chronic myeloid leukemia. Oncotarget. 2016; 7:42683-42697. http://doi.org/10.18632/ oncotarget.7977.

28. Li B, Sun B, Zhu J, Zhou N, Yang Z, Gu J. Expression of RKIP in chronic myelogenous leukemia K562 cell and inhibits cell proliferation by regulating the ERK/MAPK pathway. Tumour Biol. 2014; 35:10057-10066.

29. Eisendle K, Lang A, Eibl B, Nachbaur D, Glass1 H, Fiegl M, Thaler J, Gastl G. Phenotypic and functional deficiencies of leukaemic dendritic cells from patients with chronic myeloid leukaemia. Br J Haematol. 2003; 120:63-73.

30. Mineo M, Garfield SH, Taverna S, Flugy A, De Leo G, Alessandro R, Kohn EC. Exosomes released by K562 chronic myeloid leukemia cells promote angiogenesis in a Src-dependent fashion. Angiogenesis. 2012; 15:33-45.

31. Maia V, Ortiz-Rivero S, Sanz M, Gutierrez-Berzal J, Alvarez-Fernandez I, Gutierrez-Herrero S, de Pereda JM, Porras A, Guerrero C. C3G forms complexes with Bcr$\mathrm{Abl}$ and p38alpha MAPK at the focal adhesions in chronic myeloid leukemia cells: implication in the regulation of leukemic cell adhesion. Cell Commun Signal. 2013; 11:9.

32. Gustafsson K, Jamalpour M, Trinh C, Kharas MG, Welsh M. The Src homology-2 protein Shb modulates focal adhesion kinase signaling in a BCR-ABL myeloproliferative disorder causing accelerated progression of disease. J Hematol Oncol. 2014; 7:45.

33. Du J, Liu S, He J, Liu X, Qu Y, Yan W, Fan J, Li R, Xi H, $\mathrm{Fu}$ W, Zhang C, Yang J, Hou J. MicroRNA-451 regulates stemness of side population cells via PI3K/Akt/mTOR signaling pathway in multiple myeloma. Oncotarget. 2015; 6:14993-15007. http://doi.org/10.18632/oncotarget.3802.

34. Meng YB, He X, Huang YF, Wu QN, Zhou YC, Hao DJ. Long Non-coding RNA CRNDE Promotes Multiple Myeloma Cell Growth By Suppressing MiR-451. Oncol Res. 2017; 25:1207-1214.

35. Su R, Gong JN, Chen MT, Song L, Shen C, Zhang XH, Yin XL, Ning HM, Liu B, Wang F, Ma YN, Zhao HL, Yu J, et al. c-Myc suppresses miR-451 dash, verticalYWTAZ/ AKT axis via recruiting HDAC3 in acute myeloid leukemia. Oncotarget. 2016; 7:77430-77443. http://doi.org/10.18632/ oncotarget. 12679 .

36. Whitman SP, Maharry K, Radmacher MD, Becker H, Mrozek K, Margeson D, Holland KB, Wu YZ, Schwind S, Metzeler KH, Wen J, Baer MR, Powell BL, et al. FLT3 internal tandem duplication associates with adverse outcome 
and gene- and microRNA-expression signatures in patients 60 years of age or older with primary cytogenetically normal acute myeloid leukemia: a Cancer and Leukemia Group B study. Blood. 2010; 116:3622-3626.

37. Li X, Sanda T, Look AT, Novina CD, von Boehmer H. Repression of tumor suppressor miR-451 is essential for NOTCH1-induced oncogenesis in T-ALL. J Exp Med. 2011; 208:663-675.

38. Avigad S, Verly IR, Lebel A, Kordi O, Shichrur K, Ohali A, Hameiri-Grossman M, Kaspers GJ, Cloos J, Fronkova E, Trka J, Luria D, Kodman Y, et al. miR expression profiling at diagnosis predicts relapse in pediatric precursor B-cell acute lymphoblastic leukemia. Genes Chromosomes Cancer. 2016; 55:328-339.

39. Merkerova MD, Krejcik Z, Belickova M, Hrustincova A, Klema J, Stara E, Zemanova Z, Michalova K, Cermak J, Jonasova A. Genome-wide miRNA profiling in myelodysplastic syndrome with $\operatorname{del}(5 q)$ treated with lenalidomide. Eur J Haematol. 2015; 95:35-43.

40. Bruchova H, Yoon D, Agarwal AM, Mendell J, Prchal JT. Regulated expression of microRNAs in normal and polycythemia vera erythropoiesis. Exp Hematol. 2007; 35:1657-1667.

41. Rasmussen KD, Simmini S, Abreu-Goodger C, Bartonicek N, Di Giacomo M, Bilbao-Cortes D, Horos R, Von Lindern M, Enright AJ, O'Carroll D. The miR-144/451 locus is required for erythroid homeostasis. J Exp Med. 2010; 207:1351-1358.

42. Svasti S, Masaki S, Penglong T, Abe Y, Winichagoon P, Fucharoen S, Umemura T. Expression of microRNA-451 in normal and thalassemic erythropoiesis. Ann Hematol. 2010; 89:953-958.
43. Redova M, Poprach A, Nekvindova J, Iliev R, Radova L, Lakomy R, Svoboda M, Vyzula R, Slaby O. Circulating miR-378 and miR-451 in serum are potential biomarkers for renal cell carcinoma. J Transl Med. 2012; 10:55.

44. Al-Khanbashi M, Caramuta S, Alajmi AM, Al-Haddabi I, Al-Riyami M, Lui WO, Al-Moundhri MS. Tissue and Serum miRNA Profile in Locally Advanced Breast Cancer (LABC) in Response to Neo-Adjuvant Chemotherapy (NAC) Treatment. PLoS One. 2016; 11:e0152032.

45. Gu X, Xue JQ, Han SJ, Qian SY, Zhang WH. Circulating microRNA-451 as a predictor of resistance to neoadjuvant chemotherapy in breast cancer. Cancer Biomark. 2016; 16:395-403.

46. Jones CI, Zabolotskaya MV, King AJ, Stewart HJ, Horne GA, Chevassut TJ, Newbury SF. Identification of circulating microRNAs as diagnostic biomarkers for use in multiple myeloma. Br J Cancer. 2012; 107:1987-1996.

47. Hu Z, Dong J, Wang LE, Ma H, Liu J, Zhao Y, Tang J, Chen X, Dai J, Wei Q, Zhang C, Shen H. Serum microRNA profiling and breast cancer risk: the use of miR-484/191 as endogenous controls. Carcinogenesis. 2012; 33:828-834.

48. Qu X, Zhao M, Wu S, Yu W, Xu J, Li J, Chen L. Circulating microRNA 483-5p as a novel biomarker for diagnosis survival prediction in multiple myeloma. Med Oncol. 2014; 31:219.

49. Friedman RC, Farh KK, Burge CB, Bartel DP. Most mammalian mRNAs are conserved targets of microRNAs. Genome Res. 2009; 19:92-105.

50. Huang da W, Sherman BT, Lempicki RA. Systematic and integrative analysis of large gene lists using DAVID bioinformatics resources. Nat Protoc. 2009; 4:44-57. 